Relations industrielles

Industrial Relations

\title{
Les relations industrielles: une pratique et une discipline
}

\section{Jean Boivin}

Volume 42, numéro 1, 1987

URI : https://id.erudit.org/iderudit/050291ar

DOI : https://doi.org/10.7202/050291ar

Aller au sommaire du numéro

\section{Éditeur(s)}

Département des relations industrielles de l'Université Laval

\section{ISSN}

0034-379X (imprimé)

1703-8138 (numérique)

Découvrir la revue

\section{Citer cet article}

Boivin, J. (1987). Les relations industrielles: une pratique et une discipline. Relations industrielles / Industrial Relations, 42(1), 179-196.

https://doi.org/10.7202/050291ar
Résumé de l'article

L'auteur présente une discussion des principaux concepts utilisés en relations industrielles et examine si la façon de présenter les connaissances en ce domaine est basée sur la réalité concrète.
Tous droits réservés (C) Département des relations industrielles de l'Université Laval, 1987
Ce document est protégé par la loi sur le droit d'auteur. L'utilisation des services d'Érudit (y compris la reproduction) est assujettie à sa politique d'utilisation que vous pouvez consulter en ligne.

https://apropos.erudit.org/fr/usagers/politique-dutilisation/ 
les programmes de gestion des ressources humaines. On observe alors une forme de marchandage implicite (cryptobargaining) en vertu duquel l'employeur accorde des conditions de travail comparables à celles des entreprises syndiquées. Et si un tel processus ne favorise pas un certain équilibre, le législateur et les tribunaux interviendront davantage dans la réglementation du travail. Or de telles interventions de l'État, au nom du principe d'équité, ont aussi pour effet de limiter les pouvoirs de l'entreprise.

L'entreprise étant stimulée par le principe d'efficacité et de profitabilité, il serait inévitable, selon l'auteur, qu'un contrepoids agisse pour restaurer un équilibre relatif dans les relations industrielles. Et s'il existe des stratégies pouvant contrer l'existence du syndicalisme, aucune ne peut faire abstraction de la fonction première de cette institution, à savoir la protection de l'équité. Dans l'état actuel des connaissances, il n'est pas évident que les politiques de gestion des ressources humaines ou encore l'intervention de l'État puissent remplir cette fonction de façon plus efficace que les syndicats. Aussi l'auteur avance-t-il plusieurs raisons suggérant que le syndicalisme demeure l'institution la plus appropriée pour faire prévaloir ce principe d'équité, lequel constitue une condition nécessaire à l'efficacité. Or si les employeurs étatsuniens peuvent toujours combattre le syndicalisme pour des raisons idéologiques ou politiques, il n'est pas démontré qu'une telle position favorise l'efficacité de l'entreprise.

\title{
Les relations industrielles
}

\section{Une pratique et une discipline}

\section{Jean Boivin}

\begin{abstract}
$L$ 'auteur présente une discussion des principaux concepts utilisés en relations industrielles et examine si la façon de présenter les connaissances en ce domaine est basée sur la réalité concrète.
\end{abstract}

Les relations industrielles peuvent être considérées de façon empirique ou analytique. Sur le plan empirique, on se réfère à un univers de phénomènes qui se prêtent à une pratique ou à «l'art» des relations industrielles. Sur le plan analytique, on se réfère à l'organisation systématique des connaissances portant sur ces phénomènes et qui conduit éventuellement à la «discipline» des relations industrielles.

* BOIVIN, Jean, professeur, Département des relations industrielles, Université Laval. 


\section{LA PRATIQUE DES RELATIONS INDUSTRIELLES}

Les relations industrielles peuvent se définir, en pratique, comme «la gestion des problèmes du travail dans une société industrielle» ${ }^{1}$. Le contexte industriel moderne comprend les éléments suivants:

1- une technologie donnée;

2- des investissements importants dans les ressources humaines et matérielles;

3- une efficacité de gestion, c'est-à-dire des techniques ou moyens utilisés pour économiser sur les coûts des ressources de façon à obtenir un retour acceptable sur l'investissement;

4- une main-d'oeuvre responsable, c'est-à-dire qui a acquis une éducation, une formation et une culture la rendant sensible aux techniques d'efficacité;

5- des organisations, c'est-à-dire des regroupements structurés d'individus comprenant un réseau de règles mises en place pour faire fonctionner efficacement la technologie, les investissements et la main-d'oeuvre;

6- une bonne dose d'incertitude économique;

7- la présence de l'État qui joue un rôle régulateur et interventionniste.

La gestion des problèmes du travail implique le développement de théories, de techniques et d'institutions pour résoudre les conflits issus de la relation d'emploi. Ces conflits sont normaux dans une société industrielle et non pas pathologiques. Ils proviennent de l'interaction permanente entre l'efficacité requise par une saine gestion, le besoin de sécurité et de protection développé par les individus auxquels cette gestion s'applique et les politiques publiques développées par l'État.

On peut donc concevoir les relations industrielles comme une série d'interactions ou de processus se déroulant dans chaque organisation ainsi qu'à l'échelle de la société globale et qui peuvent être représentés selon le graphique 1.

Les éléments fondamentaux sur lesquels il importe de fournir des explications plus précises sont donc: 1) l'efficacité de gestion; 2) la communauté de travail; 3) la négociation (qui est la résultante de l'interaction entre 1 et 2 ; et 4) l'intervention et la réglementation de l'État.

\section{L'efficacité de gestion}

L'efficacité de gestion consiste dans l'organisation systématique des décisions fondamentales affectant le fonctionnement de l'entreprise (planification, direction, contrôle) en fonction d'une norme de profitabilité ou son équivalent.

1 BARBASH, J., The Elements of Industrial Relations, the University of Wisconsin Press, Madison, 1984, p. 3. Les commentaires qui suivent sur la pratique des relations industrielles sont tirés de l'introduction de ce volume, pp. 3 à 8 . 


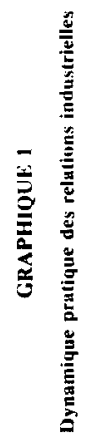

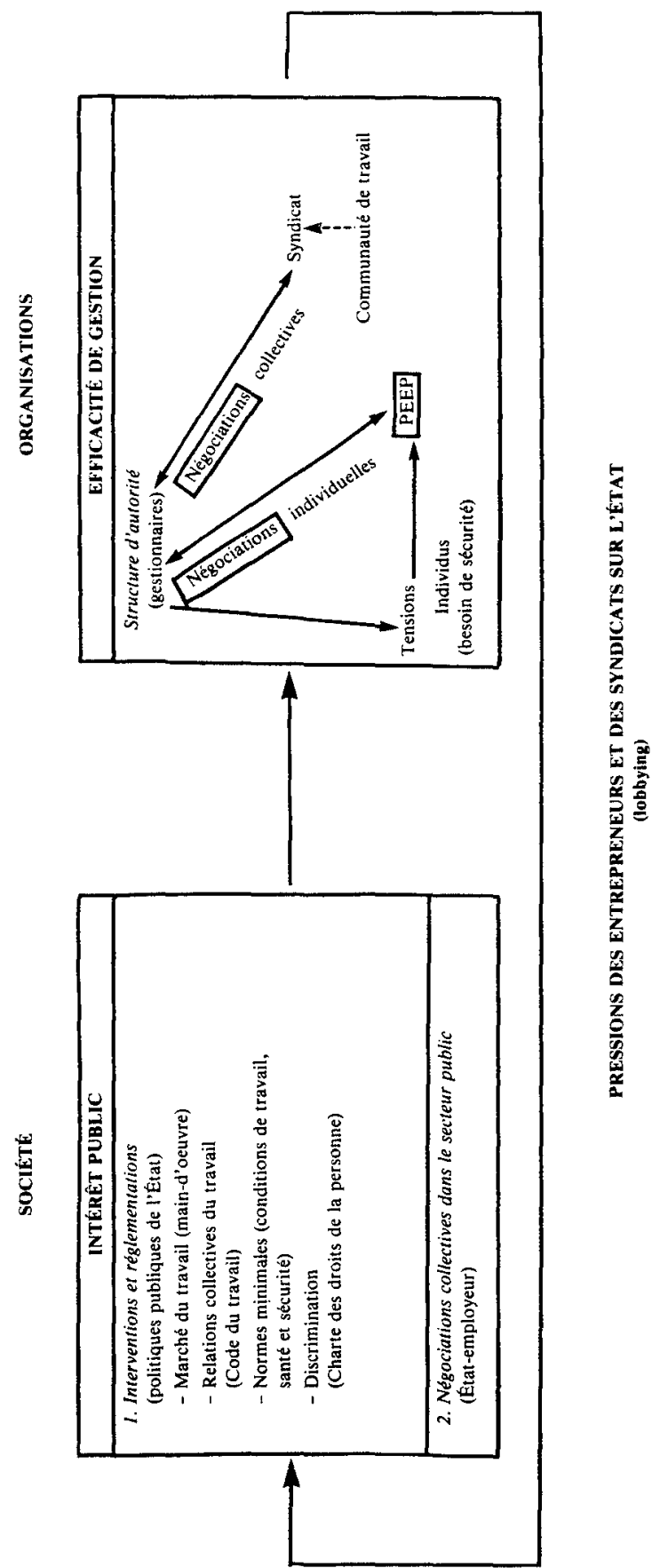


Dans le domaine plus spécifique du travail, cette efficacité de gestion comprend:

a) la professionnalisation et la spécialisation de fonctions vitales, telles l'évaluation du marché du travail et du rendement des employés, la négociation collective, la motivation des employés et

b) le développement des contrôles nécessaires pour maintenir la force de travail à l'intérieur des grands paramètres économiques de l'entreprise.

\section{La communauté de travail}

La protection collective au moyen de la communauté de travail est la réaction naturelle du travailleur face aux différentes tensions créées par l'efficacité de gestion et qui proviennent: a) de la subordination; b) de la compétitivité; c) de la monotonie et du caractère fastidieux du travail; d) de l'exploitation et e) de l'instabilité économique. Le signe "PEEP» (prix, effort, équité, pouvoir) traduit bien les intérêts particuliers qu'une communauté de travail cherche à promouvoir. En effet, la société de travail fonctionne de façon à diminuer le plus possible l'effort requis par ses membres.

Le syndicat est un type particulier de communauté de travail. C'est en fait le type le plus développé et le plus formel. Son objectif est d'agir avec efficacité comme agent négociateur et, à cet effet, il s'engage dans des activités telles le recrutement de nouveaux membres, la négociation et la grève. La prédisposition des travailleurs à se syndiquer dépend des facteurs suivants:

a) leur pouvoir de négociation individuel;

b) la menace que l'efficacité de gestion fait peser sur eux;

c) l'habileté, pour le groupe de travail, à réagir solidairement;

d) l'habileté de l'employeur à contrecarrer cette réaction;

e) les diverses façons dont la société et l'environnement économique favorisent ou découragent l'efficacité de gestion et la formation de communautés de travail;

f) les caractéristiques personnelles des travailleurs.

\section{La négociation}

La négociation est la méthode la plus souvent employée par la direction des entreprises, la communauté de travail et le syndicat pour réconcilier leurs intérêts divergents. Chaque partie se présente à la table de négociation parce qu'elle a besoin de quelque chose que l'autre partie peut lui procurer. La direction a besoin d'un travail efficace de la part de ses employés; la communauté de travail et le syndicat veulent un niveau acceptable de «PEEP» en retour de ce travail efficace. C'est généralement la perspective de perdre ce que l'autre peut lui offrir qui amène chaque partie à négocier.

La négociation est une relation ponctuée de coopération et de conflits. Les deux parties ont un intérêt commun à maximiser leurs revenus totaux, mais ils ont des intérêts divergents sur la façon dont ces revenus doivent être divisés entre les profits et les salaires. 
Les travailleurs non syndiqués peuvent souvent négocier aussi bien que les syndiqués. Même si la communauté de travail ne revêt pas le caractère formel d'un syndicat, elle peut quand même restreindre la production par l'absentéisme, les retards, l'indiscipline ou encore - par la plus forte sanction possible - le départ des travailleurs de l'entreprise. Lorsque des employés s'organisent en syndicat et refusent de travailler, on dit qu'ils se mettent en grève. La grève, latente ou réelle, est aujourd'hui le fondement de la négociation collective.

Les parties ne négocient pas seulement l'une par rapport à l'autre, mais également vis-à-vis leurs propres commettants. Elles ne sont pas monolithiques. Des intérêts divergents existent à l'intérieur de chacune d'elles et ceuxci doivent être réconciliés afin de présenter un front uni à l'autre partie.

Dans les relations patronales-syndicales où se pratique la négociation collective, la tension créée par l'opposition «efficacité vs sécurité» est partiellement résolue par une entente collective. En Amérique du Nord, ces ententes ont une durée fixe qui est généralement de deux à trois ans. De plus, l'acceptation de cette entente par la direction de l'entreprise a comme contrepartie que le syndicat s'engage à ne pas déclencher de grève pendant le temps où celle-ci est en vigueur. L'entente ou convention énumère les droits des employés et du syndicat qui sont en quelque sorte en corrélation avec le niveau de «PEEP» obtenu par la société de travail.

\section{Le rôle de l'État}

L'État participe aux relations industrielles à deux titres: soit en tant que représentant de l'intérêt public, soit en tant qu'employeur lui-même (voir graphique 1). Les domaines de l'intervention étatique sont:

1- le marché du travail, i.e. plein emploi, formation professionnelle et placement;

2- les normes minimales en matière de conditions de travail (salaires, heures de travail) et de santé et sécurité au travail;

3- les relations du travail, i.e. réglementation de la négociation collective (Code du travail);

4- le respect des droits de la personne ${ }^{2}$.

\section{Les relations industrielles dans différents pays}

Un élément important à considérer lorsque l'on compare les relations industrielles de différents pays est de connaître dans quelle mesure la négociation collective, appuyée par le recours possible ou non à la grève, est un élément décisif dans la détermination des salaires, heures de travail et condi-

2 Barbash ajoute comme domaines de l'intervention étatique: la stabilisation des prix et des salaires et la sécurité sociale. Nous croyons cependant que ces derniers se situent en dehors du champ des relations industrielles proprement dit, même si évidemment, ils l'influencent directement. Ces domaines de l'intervention étatique relèvent plutôt, selon nous, des contextes économique et social. (Voir la section sur l'approche systémique.) 
tions de travail, ainsi que dans la réglementation des conflits. À des degrés différents cependant, la négociation collective est plus importante que la réglementation étatique dans la détermination des conditions de travail en Amérique du Nord et en Europe de l'Ouest. Par contre, le rôle de l'État est beaucoup plus important dans les pays en voie de développement et dans ceux dont l'économie fonctionne selon le modèle soviétique. La négociation collective est l'apanage des régimes capitalistes socio-démocrates basés sur l'éducation de masse, le suffrage universel, l'économie de marché, la professionnalisation des relations industrielles, le pluralisme politique et l'Etatprovidence.

Les relations industrielles, dans toutes les sociétés industrielles, peu importe les systèmes politiques ou les modes de production, contiennent toutes, dans une large mesure, les éléments essentiels que sont l'efficacité de gestion et la sécurité des travailleurs.

\section{LA DISCIPLINE DES RELATIONS INDUSTRIELLES}

Plusieurs auteurs ont exprimé l'avis que les phénomènes de relations industrielles ne pouvaient pas être interprétés à partir d'une structure d'analyse rigoureuse. Ainsi, pour Neil Chamberlain «les frontières des relations industrielles sont obscures et la façon de les délimiter ne se prête pas facilement à une démarche rationnelle. Le domaine des relations industrielles s'étend à une gamme diffuse d'individus aux intérêts différents, tant aux praticiens qui ont des problèmes concrets à régler qu'aux personnes avec des préoccupations d'ordre général et plus abstraites». Pour George Shultz, «les relations industrielles sont orientées vers la solution de problèmes. Il ne s'agit pas d'une discipline en soi, mais d'un champ d'étude qui s'appuie sur plusieurs disciplines pour développer les théories et les techniques qui permettent de solutionner les problèmes de travail dans l'entreprise ou sur le marché du travail, ou encore à la table de négociation»”3.

En dépit de ces jugements pessimistes quant aux possibilités de rassembler dans un cadre d'analyse cohérent les phénomènes de relations industrielles, il y a plusieurs raisons de croire en l'utilité et en la nécessité de développer une approche plus globalisante ou plus unifiée. La première est que les relations industrielles couvrent un domaine d'activités très concrètes et portant sur des problèmes cruciaux de notre société. Il convient donc d'essayer de développer des méthodes d'analyse de ces problèmes qui permettent autant aux praticiens qu'aux chercheurs de se donner des points de référence communs, de façon à ce qu'ils puissent considérer leur travail comme faisant partie d'un ensemble plus vaste. D'ailleurs, au stade de développement scientifique actuel de la discipline, les prétentions théoriques très limitées des relations industrielles peuvent même constituer un atout intéressant: celles-ci peuvent en effet conserver un degré de généralisation assez près de la réalité pour permettre autant aux praticiens et aux chercheurs de s'y retrouver mutuellement. 
D'autre part, la raison principale qui milite en faveur d'un champ d'étude séparé pour les relations industrielles est qu'aucune autre discipline ne couvre adéquatement le territoire.

Les deux processus fondamentaux en relations industrielles: la gestion des ressources humaines et la détermination des conditions de travail

Si l'on se réfère aux deux éléments-clés qui sont à l'origine des relations industrielles dans toutes les sociétés industrialisées ou en voie de l'être, à savoir, l'efficacité de gestion et les besoins de la communauté de travail, on peut se représenter le champ d'étude ou la discipline des relations industrielles comme étant constitué de deux processus fondamentaux: un processus de gestion des ressources humaines et un processus de détermination des conditions de travail que l'on appelle ordinairement «relations du travail».

\section{La gestion des ressources humaines}

Par gestion des ressources humaines, nous entendons l'ensemble des moyens, des activités ou des programmes mis de l'avant par les organisations pour acquérir, maintenir, développer, déployer et utiliser de façon efficace les individus exerçant ou susceptibles d'exercer un travail productif.

La gestion des ressources humaines n'est pas l'apanage de l'entreprise capitaliste. Qu'elle soit socialiste, coopérative, syndicale, publique, parapublique ou privée, toute organisation a besoin de gérer des ressources humaines d'une façon efficace. De plus, la gestion des ressources humaines englobe tous les individus dans une organisation donnée, qu'ils soient cadres ou exécutants.

La gestion des ressources humaines existe également à l'échelle de la société et se traduit, en principe, par l'élaboration et l'application de politiques publiques qui visent le lieu privilégié d'actualisation des ressources humaines, à savoir le marché du travail.

Évidemment, l'application du principe d'efficacité ne revêt pas les mêmes modalités et n'implique pas les mêmes conséquences selon qu'il s'agisse d'une organisation particulière ou de la société en général. Dans le premier cas, on ne retrouve qu'une interaction entre deux agents - les gestionnaires et les exécutants - tandis que dans le second, un troisième agent est mis en cause - l'État.

Par conséquent, lorsque l'on analyse les phénomènes de relations industrielles du point de vue du principe d'efficacité, on met l'emphase sur le processus de gestion des ressources humaines. Dans cette perspective, le conflit industriel est présent, mais l'approche utilisée en est une où l'on cherche à le résorber de façon à ce que l'organisation (ou la société) atteigne ses fins. 


\section{La détermination des conditions de travail}

Le deuxième processus fondamental en relations industrielles est celui qui concerne la détermination des conditions de travail dans les organisations. Nous appelons donc «relations du travail» les phénomènes et les activités liés à l'établissement des règles au niveau du travail. Ces règles sont de deux types: les règles substantives qui déterminent les conditions dans lesquelles le travail s'effectuera (taux de salaires, durée de la journée ou de la semaine de travail, avantages sociaux, etc.), et les règles de procédure qui déterminent la façon dont on procédera pour modifier ou appliquer les règles substantives ${ }^{4}$.

Selon Clegg, il y a au moins six façons de déterminer les règles au niveau du travail. La négociation collective, même si elle est sans doute la plus connue et la plus souvent étudiée, n'est en fait que l'une de ces règles. Les autres façons de déterminer les règles régissant l'emploi sont les suivantes: 1) unilatéralement par l'employeur. Ceci est généralement le cas lorsqu'il n'y a pas de syndicat; 2) unilatéralement par le syndicat. Cette façon est moins répandue en Amérique du Nord, quoique non totalement absente, mais on la retrouve encore fréquemment en Grande-Bretagne; 3) statutairement par le législateur. C'est le cas des normes minimales; 4) en fonction des coutumes et des pratiques traditionnellement en vigueur dans les entreprises; 5) enfin, par voie de consultation de l'employeur auprès de ses salariés ou de leurs organisations, comme c'est notamment le cas dans le secteur public aux États-Unis où plusieurs employeurs, telles les municipalités, les commissions scolaires, etc., sont tenus par la loi «to meet and confer with representatives of their employees». Cette dernière méthode se situe à mi-chemin entre la négociation collective et la détermination unilatérale par l'employeur.

Cette façon d'envisager les relations du travail comme un processus d'établissement de règles fait référence à un ensemble de rapports, c'est-àdire un tout complexe d'accords, de coutumes, de pratiques, de règles et d'usages que des parties à une relation sociale acceptent comme modus vivendi et que l'État législateur tolère ou encadre. Ces accords ou ententes peuvent se faire d'individu à individu; on parle alors de relations individuelles de travail. En d'autres circonstances, les accords échapperont aux volontés individuelles pour être pris en charge, négociés et propagés par des représentants des individus; il s'agira de relations collectives du travail. Ces relations collectives, parce qu'elles s'établissent entre parties «organisées», sont la plupart du temps «institutionnalisées».

\section{Une confusion sémantique à dissiper}

Les relations du travail recouvrent plusieurs des phénomènes que l'on retrouve dans la gestion des ressources humaines. Ainsi, il va de soi que toute organisation se doit d'adopter des règles concernant l'exécution du travail, que les employés soient organisés en syndicat ou non et, en ce sens,

4 H.A. CLEGG, The Changing System of Industrial Relations in Great Britain, Oxford, Basil Blackwell,1979, pp. 1-2. 
on peut dire que les relations du travail sont un des éléments de la gestion des ressources humaines. Évidemment, dès que les employés sont représentés par un syndicat, le mode de détermination des conditions de travail est beaucoup plus "visible», car la négociation collective accapare une large part au sein de la vie de l'entreprise.

Cependant, ce qui distingue les relations du travail de la gestion des ressources humaines, c'est que chaque processus est mis en branle par l'un et l'autre des deux besoins opposés qui sont à la base même des relations industrielles: le besoin d'efficacité de l'organisation et le besoin de sécurité des membres de la communauté de travail.

Les relations du travail concernent les phénomènes visant à l'établissement du «PEEP» de Barbash, c'est-à-dire la détermination du prix (salaire et conditions de travail) que l'organisation doit payer pour récompenser l'effort physique et mental des membres de la communauté de travail qui désirent être traités équitablement et qui n'hésiteront pas à utiliser leur pouvoir pour être mieux protégés. C'est pourquoi on a souvent tendance à parler de la dynamique conflictuelle des relations de travail, puisque l'utilisation de leur pouvoir par les membres de la communauté de travail les amènera à être confrontés aux gestionnaires dont le comportement est dicté par le besoin d'efficacité de l'organisation. De plus, le "PEEP» réfère à une réalité qui s'applique également aux entreprises non syndiquées où l'initiative de déterminer les conditions de travail est laissée surtout à la direction des entreprises, quoique des «communautés de travail» plus ou moins formelles existent néanmoins, même à des niveaux élevés de la hiérarchie des entreprises (que l'on pense à l'Association des cadres supérieurs de la fonction publique).

Enfin, il tient compte de la présence de l'État puisque l'une des fonctions régulatrices de celui-ci est de s'assurer qu'un niveau minimum de «PEEP» existe au sein des organisations, surtout lorsque le pouvoir des employés est faible.

Cette très large ramification des activités concernant le «PEEP» explique sans doute pourquoi certains auteurs ont historiquement considéré comme synonymes les concepts «relations industrielles» et «relations du travail». D'autant plus que le champ d'étude appelé «relations industrielles» s'est développé parallèlement à l'institution de prédilection qui s'occupe du "PEEP», c'est-à-dire le syndicalisme. Un des plus grands analystes de ces phénomènes n'a-t-il pas d'ailleurs déclaré que la négociation collective est une institution si importante qu'elle recouvre à peu près tout le champ des relations industrielles!

Nous nous devons cependant de corriger cette affirmation et d'insister sur le fait que l'ensemble des phénomènes liés à la gestion des ressources humaines déborde les seules activités visant l'établissement d'un niveau de «PEEP» adéquat dans les organisations, même si ces dernières accaparent évidemment une large place. Prenons par exemple le cas de la sélection du personnel. Dira-t-on que les activités liées au recrutement et à la sélection du personnel (entrevues, tests, concours, etc.) ne sont pas du domaine des rela-

5 H.A. CLEGG, op. cit., p. 5. 
tions industrielles? Certainement pas. Cependant, nous serions bien surpris si même la plus vaste définition des «relations du travail» considérait la sélection du personnel comme faisant partie de son champ d'étude.

Par conséquent, alors que les relations industrielles s'intéressent à l'ensemble de la relation "efficacité $v s$ sécurité», les relations du travail n'existent qu'en fonction du second membre de cette relation. C'est pourquoi il n'est pas possible que les deux concepts soient synonymes; tout comme il est impossible de définir le tout de la même façon que l'une des parties qui le composent.

Les trois agents ou acteurs en relations industrielles

Les deux processus fondamentaux que sont la gestion des ressources humaines et les relations du travail mettent en cause trois agents ou acteurs: ce sont les organisations et leurs gestionnaires, les employés et leur communauté de travail (qui peut revêtir la forme d'un syndicat) et l'État.

Nous avons déjà suffisamment expliqué l'implication des deux premiers agents (employeurs et employés) à l'intérieur des processus de gestion des ressources humaines et de relations du travail. Il est maintenant temps d'introduire ce troisième "partenaire» dont le rôle va toujours en s'accroissant dans les relations industrielles contemporaines.

L'État, dans nos sociétés néo-libérales pluralistes, est le représentant et le défenseur des intérêts de la collectivité. Cest lui qui adopte les lois et les règles du jeu auxquelles doivent se soumettre toutes les composantes de la société. Comme principal responsable de la gestion de la société, il doit veilier à ce que toutes les ressources humaines soient utilisées efficacement, c'est-à-dire, possèdent l'emploi approprié qui convient à leurs qualifications professionnelles ainsi que des conditions de travail adéquates.

Comme nous l'avons déjà mentionné, ce rôle de gestionnaire des ressources humaines joué par l'Etat est assumé principalement par l'adoption de politiques qui visent à corriger les imperfections du marché du travail et à complémenter les grandes politiques économiques.

Mais l'État n'est pas qu'un gestionnaire de ressources humaines au niveau de l'ensemble de l'économie. En tant que responsable des intérêts de la collectivité, il doit s'assurer que les gestionnaires au niveau des organisations se comportent eux(elles)-mêmes de façon à assurer aux ressources humaines des conditions de travail adéquates. C'est pourquoi il adopte des politiques publiques visant à déterminer des conditions minimales en matière de rémunération, heures de travail, vacances, etc., ainsi qu'en matière de santé et de sécurité au travail. Certaines de ses politiques visent également à empêcher la discrimination dans l'emploi.

Un autre rôle très important assumé par l'État est celui de déterminer le cadre légal et les règles du jeu devant s'appliquer aux deux autres agents lorsque des relations collectives du travail sont établies au sein des organisations. C'est là tout le domaine de la législation du travail qui s'adresse au processus de négociation collective et à la réglementation des conflits de travail. 
Enfin, on peut considérer comme un dernier rôle, tout aussi important et distinct des autres, celui de l'aménagement de ses propres relations industrielles. L'État étant lui-même un employeur comme tous les employeurs, il doit développer un système de gestion de ressources humaines et déterminer des conditions de travail pour ses employés. Lorsque la communauté de travail de ses employés décide de recourir à l'action syndicale, il doit s'engager formellement dans des relations collectives du travail. C'est alors que surgit le dilemme «État-employeur vs État-législateur». Ce dilemme est à peu près inévitable, car en tant qu'employeur, l'État peut décider d'assujettir ses relations collectives du travail à la même réglementation que n'importe lequel autre employeur, alors qu'en tant que législateur, il peut en tout temps décider de suspendre, modifier ou ignorer cette réglementation, au nom des intérêts de la collectivitét.

Malgré tout, même si l'État représente le pouvoir ultime dans la société, il ne faut pas s'imaginer que son rôle en relations industrielles se limite à une intervention objective effectuée au nom du bien commun dans chacun des domaines qui viennent d'être mentionnés. Concrètement, le pouvoir étatique est exercé par des individus (les ministres et les députés élus formant le gouvernement) qui sont l'objet de pressions continuelles de la part de différents groupes d'intérêts (dont les entreprises et les syndicats) et qui ont leurs propres intérêts à défendre, ne serait-ce que celui de se faire réélire. Les décisions de l'État qui prennent la forme de politiques publiques sont donc adoptées en tenant compte de ce contexte particulier et, en fin de compte, ce sont les répercussions électorales potentielles qui en influencent souvent l'orientation.

Le lobbying et l'action politique exercés par les deux autres agents sont donc des activités aussi importantes que la négociation collective pour bien comprendre la dynamique du système des relations industrielles, car ils visent à influencer l'élaboration des politiques publiques dans le sens de leurs intérêts particuliers, peu importe que ces politiques touchent les marchés du travail, les normes minimales, ou le fonctionnement des règles du jeu de la négociation collective.

\section{Les trois domaines d'étude des relations industrielles}

Les deux processus fondamentaux que sont la gestion des ressources humaines et la détermination des conditions de travail mettant en interaction les trois agents que sont les organisations, les employés et l'État nous amènent à considérer les relations industrielles comme comprenant trois domaines d'étude, à savoir: la gestion des ressources humaines (GRH), les relations du travail (RLT) et les politiques publiques en matière de travail (PPMT).

Parler de trois domaines d'étude ne veut pas dire que tous les phénomènes observés en relations industrielles doivent être catégorisés obligatoire-

6 Ceci est d'autant plus vrai dans les démocraties parlementaires de type britannique où le pouvoir législatif est contrôlé par le pouvoir exécutif, sauf dans les cas de gouvernements minoritaires. 
ment dans l'un ou l'autre domaine. Ainsi, on n'a pas à se demander si un programme d'amélioration de la qualité de vie au travail (QVT) (ou encore un enseignement sur la QVT dans le cadre d'un programme d'étude en relations industrielles) constitue en soi une activité de gestion de ressources humaines, de relations du travail, ou une politique publique en matière de travail. Ce qu'il faut considérer, c'est la perspective ou l'approche avec laquelle le programme (ou l'enseignement) est offert. Si la perspective est celle de l'entreprise, on la considère comme une activité de gestion de ressources humaines; si la perspective est celle du syndicat qui désire en faire un objet de négociation collective, ce sera évidemment une activité de relations du travail; enfin, si c'est l'État qui désire mettre de tels programmes à la disposition des entreprises, on dira qu'il s'agit là d'une politique publique en matière de travail.

La distinction entre les trois domaines (GRH, RLT et PPMT) peut donc être parfois bien difficile à établir, et c'est la raison pour laquelle il ne faut pas considérer cette subdivision fonctionnelle des relations industrielles comme un guide méthodologique d'une précision infaillible. Il s'agit tout au plus d'un outil de référence utile permettant de saisir que les phénomènes de relations industrielles sont initiés, soit par des organisations soucieuses de gérer efficacement leur personnel, soit par des communautés de travail désireuses de se doter des meilleures conditions de travail possibles, soit par la puissance publique qui peut avoir des motivations très diversifiées pour intervenir.

De plus, dans le cas des politiques publiques, il peut être tout aussi difficile de distinguer celles qui relèvent du contexte des relations industrielles («en matière de travail»), de celles relevant d'autres contextes (tels économique, social, éducationnel, légal, culturel). Un critère utile, mais encore une fois non infaillible, pour faire la distinction entre une politique publique en matière de travail et les autres politiques publiques, est de vérifier si la politique étudiée est conçue dans ou à propos d'une situation de travail, ou si ce ne sont que ses effets qui touchent le travail. Car, à la limite, on pourrait considérer que presque toutes les politiques publiques sont susceptibles d'avoir des conséquences sur le travail. Par exemple, une hausse des taux d'intérêt (politique économique) provoquera un ralentissement des investissements qui, à son tour, aura une influence négative sur l'activité économique. L'activité économique réduite entraînera une hausse des inventaires qui se traduira par des mises à pied, d'où la nécessité pour l'Etat de mettre en branle des politiques de main-d'oeuvre (du domaine des relations industrielles), sans compter les plus grandes difficultés occasionnées aux syndicats qui chercheront à négocier des clauses de sécurité d'emploi. En soi, cependant, une hausse des taux d'intérêt ne peut pas être considérée comme une politique publique en matière de travail, parce qu'elle n'origine pas d'un besoin diagnostiqué sur le marché du travail, mais plutôt d'un besoin touchant l'ensemble de la société (comme la lutte à l'inflation).

Règle générale, les politiques publiques en matière de travail sont directes et sélectives, alors que les autres politiques sont globales. De plus, alors que les premières portent surtout sur le court et le moyen terme, les secondes 


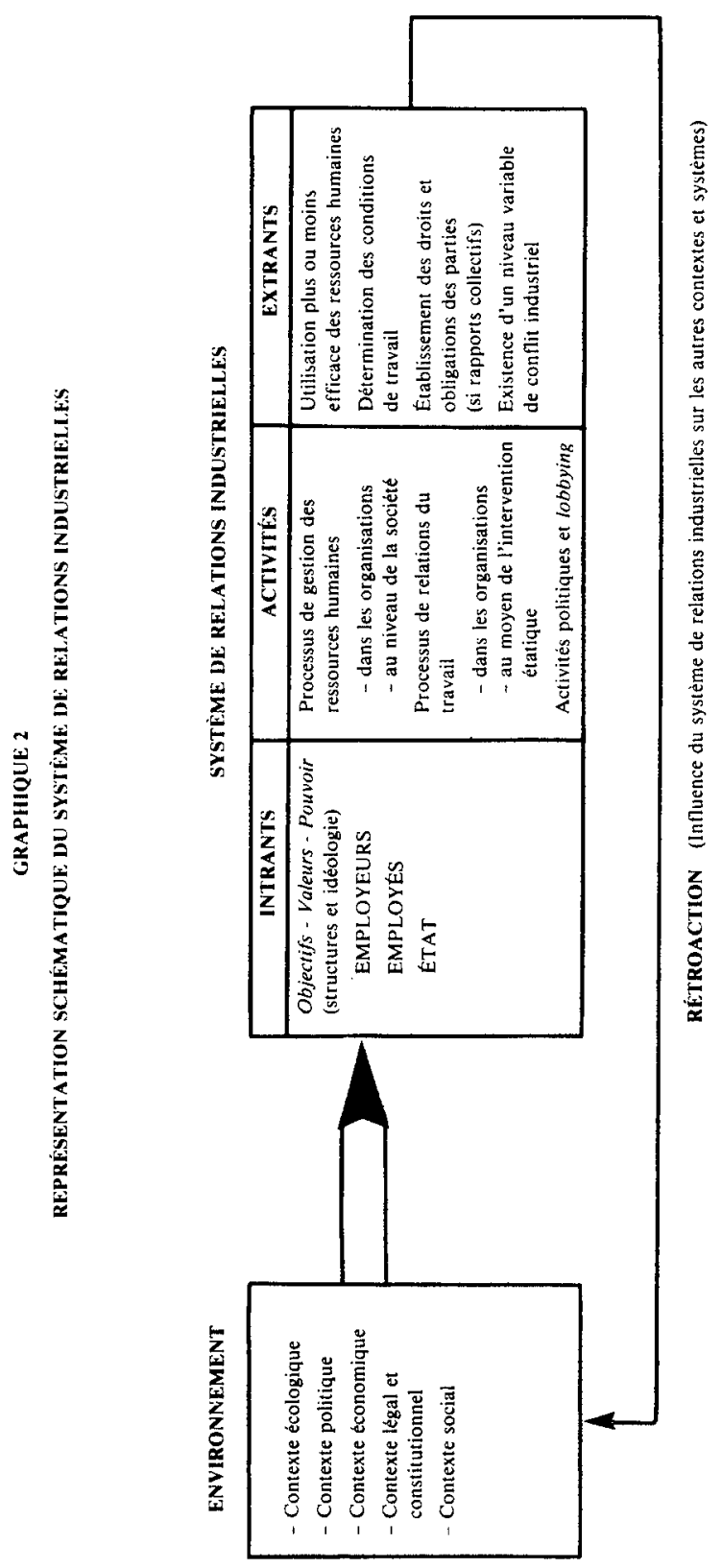




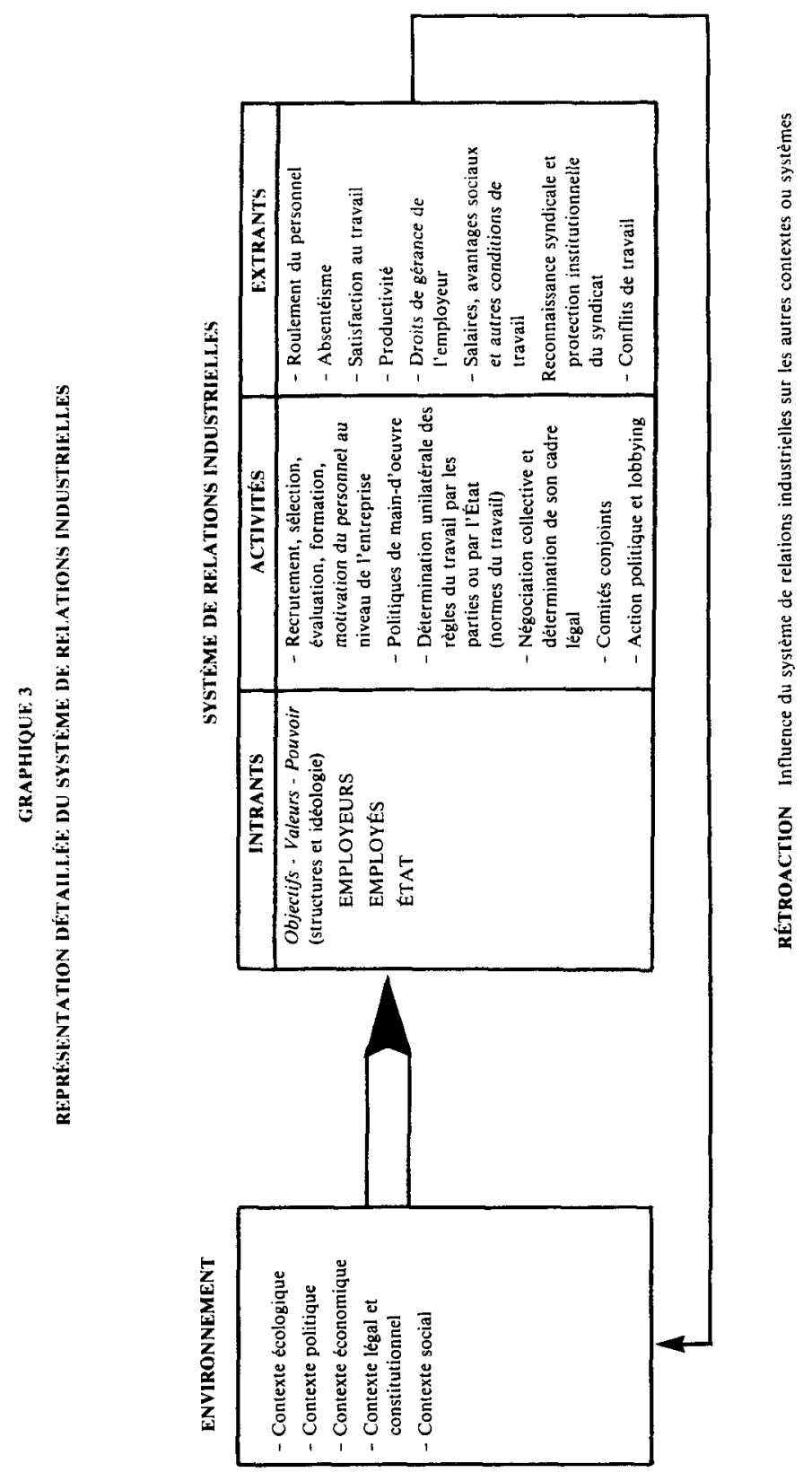


ont des effets qui se font sentir à plus long terme et, comme nous l'avons vu, de façon uniquement indirecte sur le travail?

\section{L'approche systémique}

Toute discipline qui aspire au titre de science doit se situer par rapport au concept-clé de «système». Comme le soulignent Larouche et Déom, «les sciences ou disciplines récentes (informatique, communication, service social, robotique...) de même que les plus anciennes (chimie, biologie, mathématiques, physique...) se sont «mises» à l'approche systémique en vue d'obtenir une certaine intégration des connaissances propres à leur objet d'étude et développer une certaine capacité de prédire les variations de l'objet qui leur est propre ${ }^{8}$. Les relations industrielles n'ont évidemment pas échappé à ce courant.

Si l'on définit un système comme un ensemble d'éléments différenciés et interdépendants où chacun contribue à la réalisation de l'équilibre du système, l'approche systémique doit donc permettre d'intégrer et d'organiser les connaissances propres aux objets de la discipline des relations industrielles.

La plupart des auteurs qui ont appliqué l'analyse systémique à l'étude des relations industrielles se sont presqu'exclusivement arrêtés à la dimension détermination des conditions de travail ou relations du travail de ce champ d'étude'. Nous croyons qu'une utilisation plus rigoureuse de l'approche systémique devrait également incorporer les variables liées à la gestion des ressources humaines, tant au niveau de la société qu'à celui des organisations. C'est pourquoi il nous est apparu plus conforme à la réalité de présenter le système des relations industrielles comme nous l'avons fait aux graphiques 2 et 3 .

\section{Explication des graphiques 2 et 3}

Toute société peut être conçue comme comportant plusieurs «systèmes» dont un système de relations industrielles. Les autres systèmes sont les suivants: le contexte écologique (ressources naturelles, géographie, climat); le contexte ou système économique (marché du produit, marché du travail,

7 Pour une discussion plus détaillée sur ce sujet, voir J. SEXTON, C. LECLERC, et E. DÉOM, «Politique de main-d'oeuvre et politiques publiques», Relations industrielles, les Presses de l'Université Laval, Québec, 1980, vol. 35, no 1, pp. 3 à 17.

8 V. LAROUCHE et E. DÉOM, «L'approche systémique en relations industrielles», Relations industrielles, les Presses de l'Université Laval, Québec, 1984, vol. 39, no 1, pp. 114-143.

9 John T. DUNLOP, Industrial Relations Systems, Holt, New York, 1958; Alton W.J. CRAIG, The System of Industrial Relations in Canada, Prentice-Hall, Scarborough, 1983; John ANDERSON, Morley GUNDERSON, Union-Management Relations in Canada, Addison Wesley, Don Mills, 1982; John Crispo, The Canadian Industrial Relations System, McGraw-Hill, Toronto, 1978; H.C. JAIN, Canadian Labour and Industrial Relations, McGraw-Hill, Toronto, 1975; S.M.A. HAMEED, Canadian Industrial Relations, Butterworth and Co., Toronto, 1975. 
marché monétaire, technologie); le système politique (pouvoirs législatifs et exécutifs); le système légal; le système social (structures sociales, pressions de l'opinion publique). On peut dire que l'ensemble de ces autres systèmes constitue l'environnement dans lequel «baigne» le système de relations industrielles. Cet environnement a donc une influence directe sur les intrants du système de relations industrielles.

Le système de relations industrielles met en présence les trois agents que nous avons déjà identifiés. Ce sont les intrants du système qui subissent l'influence des différents contextes environnementaux. Chacun de ces agents possède des objectifs, un système de valeurs, de même qu'un certain degré de pouvoir qui lui permettent de se structurer et de se doter d'une philosophie propre.

L'interaction de ces trois agents donne lieu à la mise en place des deux processus fondamentaux que sont la gestion des ressources humaines et les relations du travail et que l'on peut considérer comme deux types d'activités permettant de convertir les intrants en extrants ou encore, de transformer les variables indépendantes en variables dépendantes. Les processus de gestion de ressources humaines et de relations du travail sont donc considérés comme des variables intervenantes ou modératrices.

Parmi les éléments à considérer dans le processus de gestion des ressources humaines, on retrouve autant les mécanismes mis en place par les organisations pour acquérir, maintenir, développer, déployer et utiliser de façon efficace les individus exerçant ou susceptibles d'exercer un travail productif (recrutement, sélection, formation, motivation et participation des employés, création d'un climat de travail, etc.) que ceux mis en place par l'État pour faciliter le fonctionnement du marché du travail (services aux chômeurs, formation professionnelle, subventions aux entreprises pour créer ou maintenir l'emploi, etc.)

Parmi les éléments à considérer dans le processus de relations du travail, on retrouve évidemment la négociation collective et tout ce qui l'entoure, tant du point de vue des parties elles-mêmes que de celui de l'État, mais également toutes les autres façons de déterminer les règles régissant l'emploi, c'est-à-dire: 1) unilatéralement par l'employeur; 2) unilatéralement par le syndicat; 3 ) statutairement par le législateur; 4) en fonction des coutumes et pratiques en vigueur dans les entreprises; 5) enfin, par voie de consultation de l'employeur auprès de ses salariés et de leurs organisations.

Aux deux processus fondamentaux, il faut rajouter, comme mécanismes de conversion des intrants en extrants, les activités de lobbying et d'action politique qui sont utilisées par les employeurs et les employés organisés pour tenter d'influencer les décisions de l'État dans le sens de leurs intérêts respectifs.

Enfin, les variables dépendantes ou extrants sont les éléments liés aux deux processus fondamentaux qui doivent être expliqués par le système de relations industrielles, notamment le roulement du personnel, l'absentéisme, les attitudes des travailleurs, la productivité et les droits de gérance de l'entreprise en matière de gestion de ressources humaines; les salaires, avantages sociaux et autres conditions de travail, de même que la reconnaissance syndicale, la protection institutionnelle du syndicat et les conflits du travail. 
On remarquera que parmi les extrants, on retrouve des éléments qui concernent les individus tandis que d'autres concernent les organisations syndicales ou patronales.

Lorsque les extrants sont déterminés à un moment donné dans le temps, le processus de rétroaction ou la causalité circulaire des relations fait en sorte que ces extrants agissent comme des variables indépendantes qui influencent à leur tour les variables de l'environnement, et ainsi de suite.

\section{CONCLUSION}

La principale contribution de cette analyse est de fournir les justifications nécessaires pour une définition plus large du concept relations industrielles qui ne doit pas référer uniquement aux mécanismes visant à déterminer les conditions de travail mais doit aussi tenir compte de la dimension «gestion des ressources humaines», tant au niveau des entreprises qu'à celui de la société. Malgré la place considérable qu'occupe la négociation collective en relations industrielles, il n'est pas vrai que les relations collectives du travail épuisent à elles seules toutes les dimensions de ce vaste champ d'étude. Il importe donc de modifier en conséquence le modèle systémique, principal outil d'analyse permettant de regrouper les phénomènes observés.

\section{Industrial Relations A Field and a Discipline}

Industrial relations can be defined as the management of labour problems in an industrial society. Implicit in such management is the development of theories, techniques, and institutions to resolve the conflicts arising from work relations. These conflicts result from the permanent interaction of management efficiency, worker protectivism and the public policies developed by the State. The two processes that come into play because of this inevitable interaction between management efficiency (whether at the level of the organization or society in general) and the need for worker protectivism are human resource management and the establishment of working conditions, which in North America is called «labour relations».

By «human resource management» we mean all the activities or programs promoted by organizations and the State to acquire, maintain, develop, deploy and use effectively the persons doing or susceptible of doing useful work. And by «labour relations» we mean all the phenomena and activities related to the establishment of the rules for work. These rules are of two types: the substantive rules, which determine working conditions and the procedural rules, which determine the steps to be taken to change or apply the substantive rules. 
These two basic processes bring together three agents: the organization and its managers, the employees and their work society (union), and the State. The latter is involved in human resource management through its policies which seek to correct the imbalances in the labour market. It also adopts policies for the purpose of establishing minimum conditions for wages, hours of work, health, safety, and job discrimination. The State also determines the legal framework and the rules for the two other agents. Finally, since the State itself is an employer, it must like other employers develop a human resource management system and set the working conditions for its own employees.

If the empirical presentation developed previously is now examined from an analytical or academic viewpoint, we see that industrial relations include three areas of study: human resource management, labour relations, and public policies on work. Also, when the systems approach is applied to industrial relations, each of the agents is seen to have goals, values and even a certain degree of power, which allow them to organize, and to evolve their own philosophies. The interaction of the three gives rise to two types of activity that convert «inputs» to "outputs». Among the «outputs» are the turnover of personnel, absenteeism, worker attitudes, productivity, management rights, working conditions, and conflicts.

\title{
Industrial Relations Theory and Practice A Note
} Oliver Clarke

\author{
After having noted the mutual incomprehension between \\ theoritician and practitioners, the author proposes specific areas \\ on which useful work could be completed.
}

The thirst for knowledge and understanding is generally considered one of the more admirable characteristics of mankind. It is by no means evenly distributed. Those who possess it most strongly - or who are possessed by it - tend to seek jobs in research, to write, or to teach. The great majority of people, however, spend their working lives in management, production, maintenance work, administration, or the provision of day-to-day services.

* ClARKE, O., Principal Administrator, Social Affair and Industrial Relations Division, OECD, Paris.

** The views expressed in this paper are those of the author and not necessarily those of the OECD.

*** This papier is based on a presentation made to the Working Group of the International Industrial Relations Association on Industrial Relations as a Field and Industrial Relations Theory, under the Chairmanship of Professor Jack Barbash. 